\title{
Interactive Fractal Tree Generation Method having Multiple Clipping Volumes
}

\author{
SangHyun Seo, YongJun Kim and HunJoo Lee \\ Electronics and Telecommunications Research Institute (ETRI) \\ \{shseo,yj.kim,hjoo\}@etri.re.kr
}

\begin{abstract}
The Environmentally sensitive L-system expresses the fhapes of topiary by "Query module". This L-system is hard to model the tree that have multilevel grovth domain, because this system confine limited the growth using a unit volume. We extended environmentally sensitive L-system for multilevel and multimodal topiary and added the rule of main-branch and growth-area-variable in this paper. If the main-branch moves into another domain in the growing process, it is branched with the new domain as its growth-limitation area. Growtharea variable is used for checking area. We glso designednew L-system for leaves. This designed L-system generated various shaped leaves from same rule by changing parameter value. The proposed tree modelling technique is possible to model various sort of garden tree. The proposed tree modelling technique is possible to model multilevel and multimodal tree.
\end{abstract}

Keywords: Fractal Tree Modeling, Leaf Modeling, L-system

\section{Introduction}

One of the main research themes in computer graphics is modeling of natural objects. To model the natural phenomenon, fractal modeling, the particle system and grammar based modeling technique are commonly used [2-4]. Among them, grammar-based modeling technique is used to simalate the plant shape and its growth process. The most popular grammar based modeling method is L-system [7-9]. On the assumption that the plants interacts their environment, dynamic tree modeling method called environmentally sensitive L-system, has been designed to model the tree [5]. In this system, growth point (branching point) of the trées is assigned as a parameter to Query Module in each iteration step. These parameters are ysed to control the growth area dynamically. However, EnvironmentallySensitive L-system is limited to model the tree that has a multi-modal or multi-level growth area

We added main-branch production rules and growth-area-variables to previous L-system for interactive tree modeling system. In proposed L-system, when the main-branch moves from initial domain to another domain in the process of tree growth, main-branch production rule for other domain is initiated in concurrent with the branching rule for initial domain. Both rules are applied for each domain simultaneously. Growth-area-variable is used to prune the branches of tree. We could generate multi-level tree and multi-modal tree. We also propose the L-system for modeling the various leaf shapes. By combining the two L-system for the branch and leaf, we can model the final tree having multiple clipping volumes. 


\section{Previous Work}

L-system and Turtle interpreter: The L-system is called "grammar based modelling" because it produces the model by converting the string by the production rules defined in the initial step. The L-system [9] can further be subdivided into context-free L-system, contextsensitive L-system, stochastic L-system and the parametric L-system. Others include nonpropagation L-system, L-system with cut symbol and environmentally sensitive L-system. The environmentally sensitive L-system uses a query module, which stores the branching position that occurs during the growth process. These stored positions are used to control tree growth.

Stochastic tree model: Many simple models of branching structures produce ${ }^{\bullet}$ an exponentially increasing number of branch segments. Borchert and Slade shpwed hat in reality this exponential increase is not sustained beyond the early stages of tree development [6]. As soon as a tree surpasses a certain, relatively small size, the fate of branching decreases.

Environmentally sensitive L-system: In conventional L-system, the turtle interpreter interprets the string after the production rule has been applied and converts its static model into 3D data without affecting the L-system. However in environmentally sensitive L-system, the generated string is interpreted after each derivation step and tyrtle attributes found during the interpretation are returned as parameters toreserved querymodules in the string [5].

We extend our previous research [10] and enhanced the L-system that can modify and edit the clipping volume more interactivelyeang also design the L-system in order to model the leaf.

\section{Fractal Tree with Multiple ClippingVolume}

\subsection{Concepts for modeling with multipleclipping volumes}

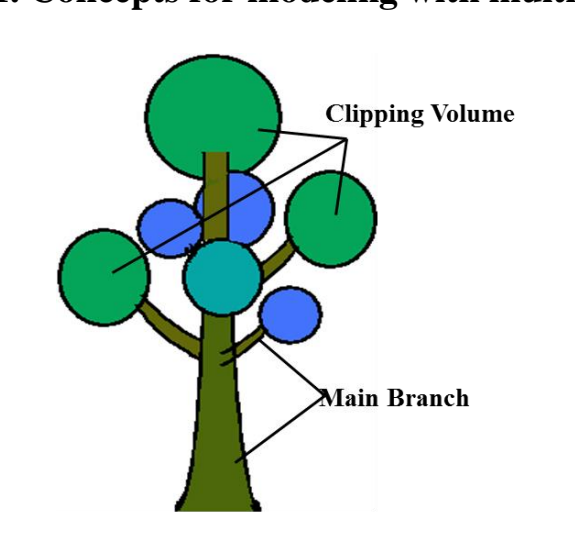

(a)

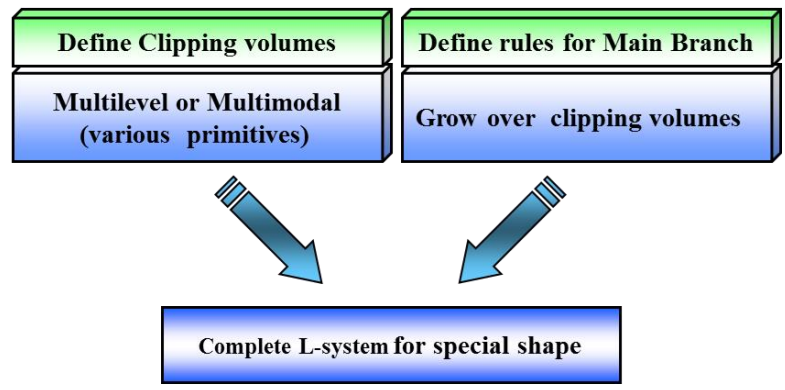

(b)

Figure 1. Preprocess for modeling the special shaped tree

A garden tree shown in Figure 1-(a) is among the trees that can be observed in the garden. It is not so easy to represent various topiary shapes using existing modeling procedures. For the multilevel or multimodal topiary shape, we add the production rule for main-branch that grows through each clipping volume and creates growth-area-variables that differentiate each clipping volume. When a main-branch grows over a clipping volume, it tests the boundary of its selected volume. The clipping volume is controlled by the growth-area-variable. Preprocess is necessary for proposed L-system. This preprocessing involves two main areas: main-branch procedure and clipping-volume procedure as shown in Figure 1-(b). Clipping 
volumes are defined by various primitives in accordance with the shapes of the trees and are assigned by an area index that distinguishes each volume. Also, main branch rule of axiom is designed in order to enable the main branch to migrate to the clipping volume. Lateral branching can be simulated by stochastic tree model [6].

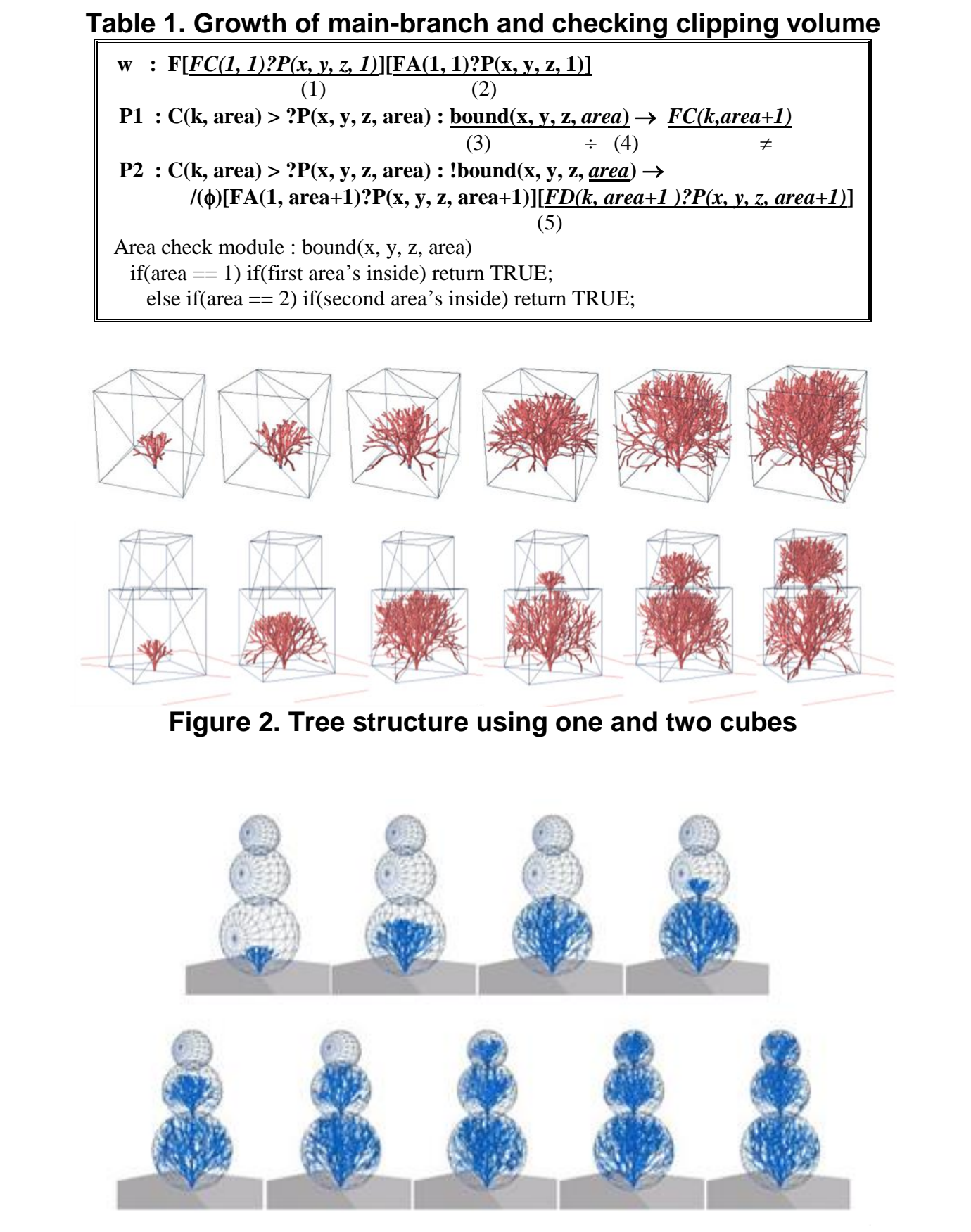

Figure 3. Tree structure using three spheres

The Table 1 shows L-system simplified by extended concept. According to axiom w, the development begins with main-branch and lateral branch. (1) consists of $F C(1,1)$, which means the beginning of main-branch growth and a query module, ?P(x,y,z,1). The symbol $A$ 
and query module in (2) shows branching of lateral branch using stochastic tree model [6]. The number 1 means the first clipping volume. (1) is transformed into productions $P 1$ or $P 2$. Production $P 1$ is the main-branch's growth. $P 2$ means lateral branch and main branch growth to new volume. The area-check-module, (3) determines whether the current position is inside or outside of the current clipping volume. And (4) of production $P l$ depicts the growth of the main-branch when the position of the main-branch is inside the first clipping volume. In this step, if the position of the main-branch is inside the second volume, $P 2$ is applied. Finally (5) changes the growth-area-variable to make the main-branch grow into the next volume. As a same approach, (3)-(5) rules can be extended for defining over two volumes. Figure 2 shows the branching pattern of the bi-m tree growing process. Figure 3 shows the shapes of trees and growth procedure using the various boundaries.

\subsection{L-system for Leaf}

The type of leaf is also various. We also introduce the paramêtric L-system for modeling the leaf in this paper. We make a hypothesis that the strycture of leaf is symmetric by $\mathrm{m}$ vein of leaf and then design the L-system. Table 2 is snom the proposed/L-system for leaf. . According to axiom w, the leaf is modelled with both sides, $L(n)$ and $R(n)$. $L(n)$ represents the left veins with main vein as its center. $R(n)$ has an opposite direction with $L(n)$. $\alpha$ means rotation angle of vein and $\beta$ represent bending angle of eachevein and offset means minimum length of vein that disturb abnormal growth of vein. Fun() can be general mathematical linear functions and control the length of each vein by function value according to the parameter, $n$. Figure 4 shows the various leaf shape by proposed L-system.

Table 2. Parametric L-system for leaf and its description

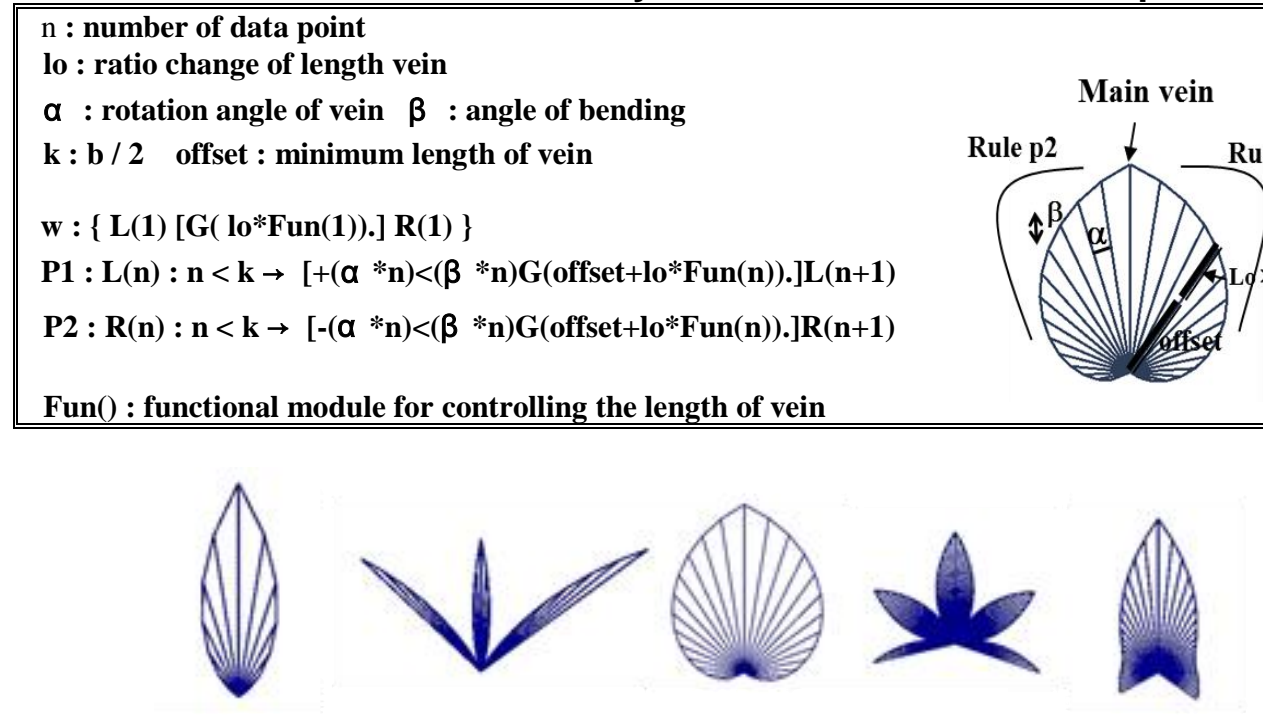

Figure 4. Leaf structure by proposed L-system

\section{Conclusions and Future work}

We propose the extended environmentally sensitive L-system, which represents tree branches with multilevel and multimodal clipping volume. In order to model the garden tree structures, we proposed the rule of main-branch and growth-area-variable in our L-system. If the main-branch moves one clipping volume into another in the growing process, branching in both volumes take place simultaneously. Growth-area-variable is used to control the volume checking. We also designed new parametric L-system for leaves. This designed L- 
system generated various shaped leaves from same rule by changing parameter value. Our modeling system provides the UI to organize the clipping volumes interactively. The proposed tree modeling technique makes it is possible to model multilevel and multimodal topiary. Figure 5 shows images that are developed by proposed L-system. In our system, the final data is stored as polygonal model. We used the ray-tracing algorithm to render the final image [1]. Our works lead to the development of interactive application program with userinput of main branch and clipping volume. We can generate the leaf type by changing the parameters of L-system for leaf modeling as well.

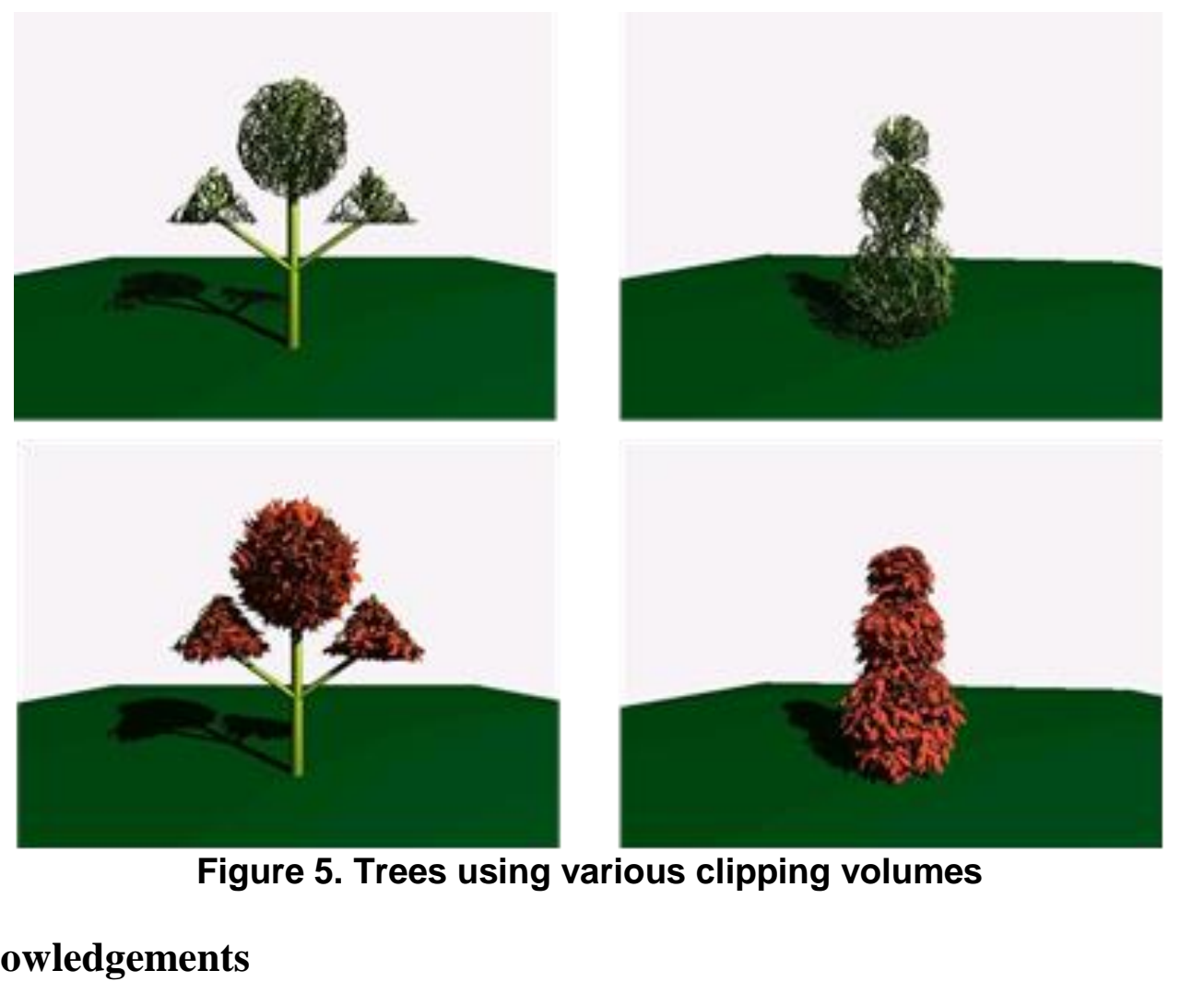

\section{Acknowledgements}

This work was supported by Ministry of Culture, Sports and Tourism (MCST) and Korea Creative Content Agency (KOCCA) in the Culture Technology (CT) and Research Development Program 2013.

\section{References}

[1] J. Foley, A. Van Dam, S. Feiner and J. Hughes, "Computer Graphics: Principles and practice”, Addison Wesley Professional (1990).

[2] M. Okabe, S. Owada, and T. Igarashi, "Interactive Design of Botanical Trees using Freehand Sketches and Example-based Editing”, Proceedings of Eurographics, (2005), pp.487-796.

[3] A. Suyantohadi, Alfiyan, M. Hariadi and M. H. Purnomo, "Plant Growth modeling using L-system approach and it's visualization", Makra Journal of Technology Series, vol.14, no. 2,(2010), pp. 92-96.

[4] O. Št'ava, B. Beneš, R. Měch, D. G. Aliaga, and P. Krištof, "Inverse Procedural Modeling by Automatic Generation of L-systems", Computer Graphics Forum, vol. 29, no. 2,(2010), pp. 665-674.

[5] P. Prusinkiewicz, M. James and R. Mech, "Synthetic topiary", Proceedings of SIGGRAPH '94, (1994), pp. 351-358

[6] R. Borchert and N. Slade, "Bifurcation ratios and the adaptive geometry of trees", Botanical Gazette, vol. 142, no. 2, (1984), pp. 184-195.

[7] J. Weber and J. Penn, "Creation and Rendering of Realistic Trees", Proceedings of SIGGRAPH '95, (1995), pp. 119-128. 
[8] L. Streit, P. Federl, and M. C. Sousa," Modelling plant variation through growth", Computer Graphics Forum, vol. 24, no. 3,(2005), pp. C487-C596.

[9] P. Prusinkiewicz, M. Hammel, J. Hanan and R. Mech, "Visual models of plant development", Spriger Verlag,(1996).

[10] S. H. Seo, Y. J. Kim and H. J. Lee, "Multi-Modal Garden Tree Modeling For Virtual World", Advance Science and Technology Letters, vol. 39,(2013), pp. 136-139.

\section{Authors}

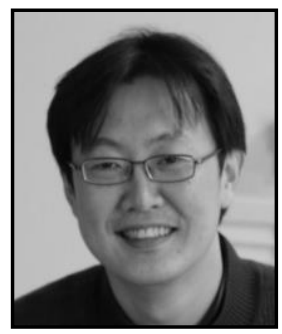

SangHyun Seo, he received his B.S. degrees in Computer Science and Engineering from Chung-Ang University, Seoul, Korea, in 1998 and M.S. and Ph.D. degrees in GSAIM Dep at Chung-Ang University in 2000 and 2010. He was senior researcher at G-Inno System from 2002 to 2005 . He was the post-doc researcher at ChungAng University, in 2010 and the post-doc reselarcher at LIRIS Lab, Lyon 1 University from Feb 2011 to Feb 2013. Now, he is working at the ETRI (Electronics and Telecommunications Research Institute), DaeJeon, Korea. He research interests are in the area of computer graphics and non-photorealistic Yendering, 3D GIS system and game technology.

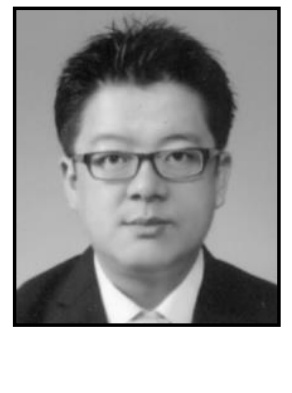

YongJun Kim, he recefyed hís B.S. in Computer Science from Hallym University, Chuncheon, Korea, in 2003. He worked at JOYMAX (Online Game Development Co.) from 2004 to 2010. He worked at)NPICSOFT (Online Game Development Co.) from 2010 to 2012. He is working at the ETRI (Electronics and Telecommunications Research Institute) since 2012. He research interests arem the area of statistical analysis and game technology.

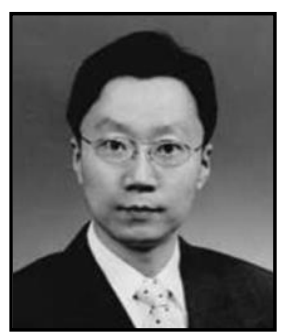

Fum Joo Lee, he received his $\mathrm{BS}$, MS, and $\mathrm{PhD}$ in computer science \& engineering from Chung-Ang University, Seoul, Rep. of Korea in 1991, 1993, and 1998, respectively. In 1998, he joined ETRI, Daejeon, Rep. of Korea. He was a postdoctoral researcher at Iowa State University, Iowa, USA, from 2001 to 2002. His current research interests include game artificial intelligence, serious game, and smart content. 